Itinéraires Itinéraires

Littérature, textes, cultures

2019-2 et $3 \mid 2019$

Corps masculins et nation : textes, images,

représentations

\title{
L'homme politique comme figure paternelle dans les films français des années 2000
}

The Male Politician as a Paternal Figure in French Cinema from the 2000's

\section{Robin Hopquin}

\section{OpenEdition}

\section{Journals}

Édition électronique

URL : http://journals.openedition.org/itineraires/6779

DOI : $10.4000 /$ itineraires.6779

ISSN : 2427-920X

Éditeur

Pléiade

Référence électronique

Robin Hopquin, «L'homme politique comme figure paternelle dans les films français des

années 2000 », Itinéraires [En ligne], 2019-2 et 3 | 2019, mis en ligne le 27 novembre 2019, consulté le

15 décembre 2019. URL : http://journals.openedition.org/itineraires/6779; DOI : 10.4000/itineraires. 6779

Ce document a été généré automatiquement le 15 décembre 2019.

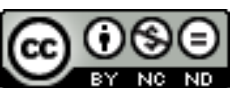

Itinéraires est mis à disposition selon les termes de la licence Creative Commons Attribution - Pas d'Utilisation Commerciale - Pas de Modification 4.0 International. 


\title{
L'homme politique comme figure paternelle dans les films français des années 2000
}

The Male Politician as a Paternal Figure in French Cinema from the 2000's

\author{
Robin Hopquin
}

\section{Introduction}

1 Depuis le début des années 2000, le cinéma français est marqué par une recrudescence de la représentation des élus, notamment des élus nationaux, sans précédent dans son histoire. S'il est possible de citer certains exemples emblématiques qui émaillent la cinématographie française, comme Le Président (Henri Verneuil, 1961) ou encore Le Bon Plaisir (Francis Girod, 1984) symptomatiques de ce que Yannick Déhée nomme «les fictions de gauche » (Déhée $2000: 152$ ) en référence à la noirceur du portrait politique de ces films, ceux-ci restent toutefois minoritaires ${ }^{1}$. C'est d'autant plus surprenant que différentes cinématographies, qu'elles soient soviétiques ou américaines, sont saturées par la représentation des élus nationaux. Faut-il comprendre, comme Jean-Michel Frodon l'avance, que "l'idée de la démocratie qu'il [le cinéma français] promeut fonctionne à l'opposé du mythe à l'américaine: en préférant les petites formes à l'épopée, la nuance au manichéisme, le singulier au général» (cité par Déhée 2000 : 172) ?

2 Si cette lecture peut être discutée - d'abord parce qu'elle présume d'une dimension seulement épique du mythe, ce qui depuis Roland Barthes au moins a largement été remis en cause, ensuite parce qu'elle valorise une vision fortement auteuriste du cinéma français - elle a le mérite de mettre en valeur une différence réelle entre un cinéma où la représentation des politiciens œuvre souvent à la construction du récit national (cinéma américain) et un autre où cette représentation, rare, ne sert pas nécessairement l'image de la nation (cinéma français). Est-ce à dire que le cinéma 
français ne s'embarrasse d'aucun lien avec le récit national? Qu'il est déconnecté de l'imaginaire national parce que plus intéressé par « la nuance » et le « singulier »?

Il est possible de dresser une liste de films représentant un ou plusieurs personnages de politiciens $^{2}$ depuis le début des années 2000: Le Promeneur du Champ-de-Mars (Robert Guédiguian, 2005), Le Candidat (Niels Arestrup, 2007), Coluche l'histoire d'un mec (Antoine De Caunes, 2008), Pater (Alain Cavalier, 2011), L'Exercice de l'État (Pierre Schoeller, 2011), La Conquête (Xavier Durringer, 2011), Les Saveurs du Palais (Christian Vincent, 2012), Quai d'Orsay (Bertrand Tavernier, 2013). Si l'on observe ces films de plus près, on remarque que beaucoup se concentrent sur le processus électoral (Le Candidat, Coluche, Pater, La Conquête), d'autres sur les rouages de la politique (L'Exercice de l'État et Quai d'Orsay), enfin, les derniers sur des figures d'hommes politiques réels ou fictifs (Le Promeneur du Champ-de-Mars et Les Saveurs du palais). Ces œuvres n'offrent que peu de récits édifiants qui permettraient un rapprochement valorisant entre le récit politique et le récit national. Plus encore, certaines cherchent même à mettre à mal des symboles de la nation, par exemple en se montrant critique envers des personnalités qui l'ont représentée (le passé collaborationniste du président du Promeneur du Champs-de-Mars qui rappelle les questions entourant celui de François Mitterrand ou le portrait acide de l'élection d'un certain Nicolas S. dans La Conquête). Dans leur ensemble, ils s'inscrivent dans une lignée qui démythifie la politique et ses représentants.

Pourtant, malgré la diversité des cinéastes, des tons et des scénarios, les films représentant des élus nationaux depuis les années 2000 se distinguent par la manière dont ils mettent en scène directement ou indirectement une figure paternelle, faisant de ce trait structurel un point d'appui privilégié pour les approcher. Ces films présentent des personnages inscrits dans la sphère politique, qu'ils soient présidents (Pater, Les Saveurs du Palais, et un ancien président dans Le Promeneur du Champs de Mars), candidats à la présidence (Le Candidat, Coluche, La Conquête) ou ministres (L'Exercice de l'État, Quai d'Orsay), où tous les postes à responsabilités sont occupés par des hommes qui incarnent une figure de paternité soit dans le cercle privé (père de famille) soit dans le cercle public (père symbolique), je reviendrai sur ce point ${ }^{3}$. Cela peut être le personnage de Coluche montré comme un père défaillant, ou les rôles maritaux tenus par Nicolas S. dans La Conquête et Michel dans Le Candidat dont les profils paternels se partagent avec une recherche de masculinité virile (expliquant par là l'absence de rôles féminins marquant, nous le verrons par la suite), ou bien encore les figures tutélaires contrastées du ministre Taillard dans Quai D'orsay, du président dans L'Exercice de l'Etat ou, exemplairement, de Alain Cavalier dans Pater, un film au titre particulièrement éloquent sur la question. Ce rapport entre la représentation du pouvoir et le père n'a rien d'anodin dans la mesure où cela dénote un lien aigu entre l'image politique telle qu'elle est présentée dans les films et l'image de la nation, même si ce lien se fait par une voie détournée, souterraine, qui est celle de la figure du père.

5 Il convient de noter à quel point le rapport avec l'image paternelle est consécutif de la construction nationale. D'abord, parce que la nation se constitue au XIXe siècle « selon un projet masculin» (Kott 2006: 168). La masculinité de l'identité nationale se remarque à deux échelons : d'abord par l'importance de la virilité (le rôle primordial de la figure guerrière et des attributs virils dans les représentations nationales démontre bien cette idée), ensuite par celle de la paternité (on le remarque par le besoin de chercher des pères fondateurs de la nation) ${ }^{4}$. Si les deux notions ne sont pas antinomiques (elles s'allient parfois dans les films), il faut admettre que le modèle 
paternel prend à rebours les représentations d'un corps masculin héroïque et viril comme référent national. Les attributs physiques du père sont moins portés en étendard qu'ils ne soulignent discrètement « l'expérience, la prudence, le sang-froid, la mesure, la modération » (Girardet $1986: 74)^{5}$. À cet égard, « le père est toujours [...] une figure symbolique, celle du pouvoir, qu'il soit naturel, divin ou au contraire fruit de la volonté des hommes » (Verjus $2013: 14)$.

6 L'image du père a une réalité dans le monde politique $d u x^{e}$ siècle. Cette dernière excède les régimes dictatoriaux où la rhétorique du père incarnée par le chef d'État trouve une expression exacerbée ${ }^{6}$. Raoul Girardet explique à quel point le mythe politique du sauveur qui caractérise certains hommes d'État (il cite Gaston Doumergue ou Charles de Gaulle), quand il ne fait pas appel aux « Pères fondateurs » de la nation (Girardet 1986: 77), se caractérise par des références à la maison ou au foyer, qui évoquent la sphère familiale dans laquelle il s'agit de restaurer l'ordre ${ }^{7}$. Plus récemment, dans une interview donnée pour le magazine Society en 2014, François Hollande, alors président de la République, définit son statut en déclarant que «le Président est le chef de la famille française » (Anese 2014:27). Il perpétue ainsi une tradition fortement ancrée dans l'imaginaire politique qui consiste à lire le statut présidentiel selon le modèle familial traditionnel, et cela depuis les débuts de la $\mathrm{V}^{\mathrm{e}}$ République dans la mesure où son instauration induit que « le rôle présidentiel devient la figure centrale du régime » où "se trouve affirmée l'autorité du chef, du père de la nation» (Achin $2008: 21$ ).

7 La correspondance entre l'apparition de films centrés sur des personnages issus du milieu politique et l'importance de modèles tutélaires associés directement ou indirectement à la paternité dans le cinéma français des années 2000 a de quoi poser question. Pour la première fois, le cinéma semble prendre en charge une représentation régulière des élus, suppléant en cela le relais incessant des images télévisuelles. On peut d'ailleurs se demander si ce n'est pas cette hyperactivité de l'image politique qui pousse les cinéastes à s'emparer d'une représentation souvent déléguée aux communicants et aux journalistes. Le statut de l'image cinématographique est particulier puisque le cinéma intervient sur un temps long, sans besoin de suivre le flux d'informations quotidien. Il est ainsi un regard sur une image déjà constituée. Représenter le pouvoir c'est filmer ce qui en soi est déjà une représentation. À ce sujet, Louis Marin explique à propos du corps du roi que «le roi n'est vraiment, c'est-à-dire monarque, que dans les images ${ }^{8}$ » (Marin 1981 : 170). À ce titre il existe un double corps du roi : l'un physique et l'autre fictionnel, l'un présent et l'autre représenté. Le roi, l'homme de pouvoir, l'élu, est à la fois lui et hors de lui, c'est-àdire que le pouvoir est avant tout une image, qu'il existe par la représentation. Ainsi, la question est de savoir si les films cités font bel et bien exister une figure de père par l'image du corps politicien. En d'autres termes, si par le corps représenté du politicien, s'incarne la «figure symbolique » du père, qui est celle du " pouvoir ».

8 Cette dualité du corps de l'homme politique est primordiale pour comprendre le positionnement des cinéastes vis-à-vis de l'image du père. La présence des figures paternelles dans les films interroge sur la persistance du mythe patriarcal de la nation à l'intérieur d'une représentation du monde politique, qu'on reconnaît à l'extrême dans le titre du film d'Alain Cavalier Pater. Il faut pourtant prendre garde à ne pas considérer cette figure comme acquise dans les films cités, dans la mesure où rien n'indique a priori qu'ils acceptent l'image du père sans la critiquer ou en dévoiler l'artifice. Ainsi, il s'agit 
de comprendre si le cinéma permet lui aussi de dédoubler ce corps de l'homme politique et de l'investir d'une dimension sacrale, qui se manifesterait par l'image du père.

9 L'objectif sera donc d'identifier dans ces films les répertoires, les lexiques et les chaînes de signification par lesquels se révèlent les contours d'un possible impensé patriarcal, dont les limites se jugeront à l'aune des œuvres étudiées. Si des écarts et des franchissements à ce modèle sont dévoilés, il faudra dans un premier temps reconnaître un facteur commun à l'ensemble des films qui permettra de cadrer les représentations de la figure du père. Ce facteur est l'absence ou la marginalisation dans les cercles du pouvoir des personnages féminins. Ce premier élément permettra de démontrer la prégnance d'une vision exclusivement masculine du pouvoir, avant de comprendre comment cette masculinité est d'abord interrogée à l'aune des rapports filiaux, définissant le pouvoir public en rapport avec les relations entre les personnages dans le cercle privé, puis questionnée selon un modèle initiatique, en faisant du père une figure symbolique de passage à l'intérieur du cercle politique. Ces deux manières de représenter le père ordonnent des regards divergents sur la figure paternelle : tout en s'appuyant sur une marginalisation de la présence féminine dans les cercles du pouvoir, on remarquera que certains films prennent le père comme un acquis, une évidence politique, alors que d'autres mettent en doute sa viabilité et travaillent à sa démythification.

\section{La place de la femme : un cinéma misogyne?}

10 Malgré la diversité des films présentés ici, le premier point commun qui les rassemble à peu près tous est la dévalorisation, voire l'absence, des personnages féminins dans la représentation des cercles du pouvoir. Cette faiblesse est d'autant plus marquante qu'elle sert quasiment exclusivement à caractériser le personnage masculin en tant que figure tutélaire. La marginalisation du personnage féminin fonde les prémices de l'installation d'une figure paternelle dans l'ensemble des films étudiés. En cela ces films peuvent se comprendre comme des moyens de légitimer systématiquement un rapport de domination homme-femme qui, tout en plaçant la femme dans des positions mineures, accrédite la vision d'un pouvoir masculin.

\section{L'invisible féminin}

11 La première tendance qui caractérise les films étudiés s'incarne dans leur propension à ne pas montrer de femmes à l'écran, ou bien d'une façon si fugace qu'elles semblent n'avoir aucun lien avec le monde qui est filmé. Pater est l'exemple le plus radical puisqu'aucun personnage féminin n'y est représenté, sinon une fois, dans une scène particulièrement évocatrice : on y voit Vincent Lindon au lit, se réveiller un matin avec une femme qui dort et nous tourne le dos, il lui caresse la peau rapidement et se lève. C'est la seule apparition féminine que propose Pater, et on ne voit pas le visage de la femme. Comme le note Geneviève Sellier (2012), la femme est assimilée au « repos du guerrier " donc au réconfort tranquille du foyer et de la famille, ceci d'autant plus facilement qu'elle n'a pas de visage, comme si on pouvait lui substituer n'importe quelle autre femme. Elle rappelle ainsi « la féminité mutique des figures allégoriques » (Achin $2008: 35$ ). Aussi impersonnel et universel, son dos nu se fait moins le symbole du 
féminin que celui de la virilité, c'est-à-dire moins une femme qu'une conquête ${ }^{9}$. Le titre du film apparait alors comme l'affirmation de cette désertion de la figure féminine : Pater, ou la vision d'un monde sans femme.

Le corps féminin montré à nu est l'occasion de définir par extension un corps masculin sacralisé dont l'habit se fait l'attribut de sa légitimité. Le corps politique est un corps secret, qu'on ne peut montrer de front, de crainte qu'il soit ramené à sa faiblesse ontologique, celle d'un corps d'homme fatigué, disgracieux.

Le seul film récent qui déshabille un homme politique semble être le film d'Abel Ferrara sur Dominique Strauss-Kahn (Welcome to New York, 2014). Après son arrestation, le politicien (Gérard Depardieu) est conduit en prison où il doit, par étapes, se déshabiller jusqu'à être nu. Cette mise à nu vient métaphoriser sa déchéance : le voile a été levé, débarrassé de ces oripeaux, il n'est qu'un homme présenté dans toute sa faiblesse. Cependant, Welcome to New York cherche justement à désacraliser la figure politique en utilisant un personnage aussi polémique que celui de Dominique Strauss-Kahn.

Hormis ce contre-exemple, le corps de l'homme politique est plus volontiers montré comme souverain. Il peut être dédoublé dans l'image, comme dans La Conquête où Nicolas S. sur l'estrade est redoublé par sa propre image projetée derrière lui, mettant ainsi en pratique ce double corps de l'homme de pouvoir que décrit Louis Marin: le corps physique du personnage d'un côté, et son corps imagé de l'autre, ici grandi par un effet de projection. L'homme de pouvoir peut aussi être isolé dans un plan et dans un décor trop large, qu'il habite pourtant seul, comme dans L'Exercice de l'État sur lequel nous reviendrons par la suite.

\section{La « femme de »}

Dans certains films, le personnage féminin se réduit simplement à être la " femme de ", son interaction avec le personnage masculin permettant seulement de caractériser ce dernier. Dans Le Candidat, Michel (Yvan Attal), qui candidate à la présidence, est d'abord décrit comme un personnage dont le couple ne se porte pas bien. Pour sa première apparition, il est seul dans la salle de bains (baignée d'une lumière bleue) à écouter la radio dans un instant de méditation, alors que sa femme s'affaire dans la pièce adjacente (baignée d'une lumière jaune). L'interaction entre Michel et Laura (Stefania Rocca) se fonde sur des oppositions esthétiques: il est dans le bleu, elle est dans le jaune; il médite, elle s'affaire (et boit); comme s'ils ne vivaient pas dans le même monde. Sur l'ensemble du film, le personnage de Laura est instrumentalisé pour décrire la progression psychologique de Michel. Dans la première partie du film, on adopte volontiers son regard car elle semble être la seule à comprendre à quelle impasse mène l'équipe de communication de Michel ; c'est de son point de vue qu'on regarde souvent ces personnages méprisables. De fait, elle fuit cette prison dorée et abandonne Michel à son sort. Plus tard dans le film, quand Michel a récupéré son indépendance en congédiant son équipe de communication et qu'il se prépare pour un débat télévisé décisif, Laura réapparait sur le pas de la porte, défaite, en bas de l'escalier. En adoptant le point de vue de Michel et en filmant ainsi la jeune femme en forte plongée en contrebas, le réalisateur accrédite à la fois la priorité accordée au regard masculin et l'aspect dérisoire de la femme. La lecture qu'on peut faire de cette réapparition souligne bien le manque d'intérêt accordé à la figure féminine : elle revient vers un homme qui a su reconquérir sa force. De fait, le film met en avant la façon dont Michel 
devient un politicien et plus pragmatiquement, comment il devient un homme. Le retour de la femme n'a pour enjeu que la caractérisation de Michel en homme qui conquiert le pouvoir. Le dernier plan dans le jardin, où Michel et Laura prennent leur petit-déjeuner au soleil, incarne l'aboutissement d'un retour au pouvoir masculin: la femme est redevenue aimante et l'homme a renoué avec sa virilité.

\section{La femme politique}

Quelle place la femme occupe-t-elle dans le travail politique ? À peu près toujours la même, elle n'est jamais au pouvoir, dans aucun des films, elle est toujours une communicante, une salariée au service d'un homme politique. Dans L'Exercice de l'État, Pauline est responsable de l'image de Saint-Jean, elle est, selon l'expression de Geneviève Sellier (2012), moins un "alter-ego " qu'une "gouvernante » qui gère les détails de l'apparence du ministre (même si, parfois, leurs relations ressemblent à celles d'un couple).

Les Saveurs du palais est un film révélateur quant à l'évolution de son personnage féminin. Hortense Laborie (jouée par Catherine Frot) est nommée cuisinière en chef des cuisines du président de la République qui ne sont peuplées que par des hommes. Christian Vincent utilise la cuisine pour critiquer en sous-texte la situation misogyne de la politique en France. Pourtant, assez rapidement, Hortense va là encore se réduire à un cliché : celui de la mère nourricière. La figure maternelle renforce d'autant plus la prégnance du chef d'État comme père, ici joué par Jean d'Ormesson. Cela tient d'ailleurs au film en lui-même où toutes les relations rappellent le modèle familial traditionnel. La mère nourrit, le père mange (le président demande à Hortense de lui faire des plats "comme sa mère les lui faisait", en l'occurrence des plats du terroir). Tout entreprenante et active qu'elle soit, Hortense ne reste qu'une version améliorée de femme au foyer, d'ailleurs moins une femme qu'un fantasme de petit garçon. On peut rapprocher ce personnage d'une figure de femme "régente", comme l'expliquent Catherine Achin et Elsa Dorlin :

on a toujours tenté de ramener les femmes politiques à leur statut de « mère ", mais de mère toute-puissante et régente avant tout : autrement dit, ce sont des femmes qui ne sont acceptées dans le cénacle du gouvernement que parce qu'elles ne représentent qu'une phase transitoire du pouvoir. (Achin $2008: 36$ )

Les Saveurs du palais est en quelque sorte l'antithèse d'un film politique, c'est pourquoi son exemple est intéressant : il tend vers la misogynie alors qu'il voudrait justement dénoncer une situation problématique, mais il est aussi réactionnaire, accroché au doux souvenir d'une France d'antan qu'il réinterprète en creux.

Dans la représentation du monde politique, la femme est donc concentrée dans deux types qui définissent sa relation par rapport à l'homme : elle est gouvernante ou mère et n'occupe pas de poste de pouvoir.

Il faut tirer deux conclusions de cette absence de figure féminine forte: d'abord, les films reproduisent un schéma social contemporain (peu de femmes au pouvoir) dont ils ne remettent pas en cause la légitimité et qu'ils reconduisent sans questionner. Ils renvoient alors à « des constructions enracinées dans l'imaginaire social, qui véhiculent le préjugé selon lequel les femmes n'ont pas les aptitudes nécessaires pour gouverner " (Liatard $2008: 8$ ). En ce sens, ils entérinent par une représentation jugée normale, une donnée sociale traditionnelle qui mériterait plutôt d'être interrogée. 
20 Deuxièmement, cette déconsidération esthétique pour la figure féminine affirme par là même la prééminence du pouvoir masculin et, plus précisément, de la figure paternelle. Le pouvoir ainsi assimilé au masculin est investi d'une dimension traditionnelle par laquelle le corps de l'homme est le seul légitime à se dédoubler dans les images du pouvoir.

\section{L'idéal familial : le mauvais père}

21 Il existe deux tendances dans les films étudiés : certains présentent seulement un personnage (principal ou non) de politicien (La Conquête, Coluche, Le Candidat), d'autres suivent un personnage de «jeune » qui entre dans le milieu politique (Pater, L'Exercice de l'État, Quai d'Orsay $)^{10}$. Cette distinction en produit une autre : les premiers films cités proposent peu de regards extérieurs sur le personnage politique, alors que les seconds sont justement l'occasion d'exercer ce regard, d'éprouver la figure politicienne au-delà de sa sphère privilégiée. Ainsi, les premiers ne font pas vivre de figure paternelle à l'intérieur du dispositif filmique (par le regard d'un plus jeune sur le politicien), au contraire des seconds, qu'on peut lire comme des récits initiatiques.

Cette distinction qui pourrait paraître bénigne construit pourtant une vision différenciée de la figure paternelle, c'est-à-dire que les premiers prennent cette donnée comme acquise (le président doit être le chef de la famille française) quand les autres la façonnent par le dispositif filmique (échange de regards entre un «fils » et un " père »). Ainsi, les films dont le personnage principal est directement le politicien et qui ne mettent pas en relief sa position par le regard d'un nouvel arrivant, un «fils" symbolique, ont une tendance à illustrer la figure paternelle par l'intermédiaire du cercle privé. Ils utilisent les relations entre personnages au sein du cercle familial pour caractériser directement une figure paternelle qui est censée pouvoir s'étendre au domaine public. En d'autres termes : un bon père caractérise un bon homme d'État ; un mauvais père, un mauvais dirigeant (le «père » et le «chef» étant placés sur un pied d'égalité).

\section{La Conquête : le père sans autorité}

La mise à niveau du cercle privé (père ou mari) et du cercle public (président ou candidat) prend forme, dans La Conquête, à partir des relations de Nicolas S. (Denis Podalydès) avec sa femme (Cécilia jouée par Florence Pernel). À partir de la relation mari/femme, Xavier Durringer décrédibilise Nicolas $S$. et sa capacité à incarner un père pour la nation. C'est par sa femme que Durringer dépeint Nicolas S. comme un petit roi, un enfant gâté plutôt qu'un père et un mari responsable.

La Conquête est un film original dans le corpus par l'intérêt qu'il porte à la relation conjugale entre Nicolas S. et Cécilia S., puisque c'est le seul qui se concentre aussi profondément sur une romance. La Conquête ne tourne d'ailleurs qu'autour de l'histoire amoureuse : alors qu'il attend les résultats des élections présidentielles, le seul souci de Nicolas $\mathrm{S}$. est de savoir où est sa femme. C'est donc une relation de dépendance que le film décrit ; Nicolas S. ne cesse d'ailleurs de demander conseil à Cécilia S., d'en faire sa première conseillère. Plus encore, son mal-être moral (déprime) et politique (chute dans les sondages) sont associés à sa rupture avec Cécilia, et son regain de forme au retour de celle-ci. 
Si elle crée de l'empathie avec Nicolas S., cette dépendance envers sa femme en fait aussi un personnage ridicule, enfantin et immature, qui sous-entend in fine une faiblesse humaine et politique, comme si cette relation de dépendance devait symboliser tout le parcours politique du personnage. Comme le note Geneviève Sellier : "le manque de légitimité dans l'exercice du pouvoir est directement mis en relation avec le manque d'autonomie du personnage par rapport à sa femme. Le film semble nous dire: "comment un homme qui se comporte avec sa femme comme un petit garçon peut-il diriger l'État ?" ». Elle ajoute : «le scénariste Patrick Rotman a préféré mettre l'accent sur la dépendance affective du personnage par rapport à une femme, comme marque d'une identité politique défaillante » (Sellier 2012). L'argument du film fonctionne presque par l'absurde en rendant le rapport entre le masculin et le pouvoir circulaire : si Nicolas S. n'est pas un bon mari, il ne peut être un bon père, et donc un bon président.

Autant qu'on y trouve, de nouveau, les traces d'une misogynie décomplexée, on s'aperçoit que les relations dans le cercle privé caractérisent l'homme public et même, au-delà, la part d'engagement politique chez lui. Comme dans Le Candidat, le modèle conjugal fixe les qualités de l'homme politique, donc du modèle politique (en bien dans Le Candidat, en mal dans La Conquête).

Dans la représentation contemporaine du politicien, les qualités traditionnelles, voire réactionnaires (dominer sa femme) sont montrées comme partie intégrante d'une capacité à gouverner, consécutives d'une aura de « chef».

\section{Coluche : I'impasse} ou plutôt comme un père absent, distant, qui laisse la charge des enfants à sa femme. Dans une scène évocatrice, Coluche sort de chez lui alors que ses deux enfants se lancent des corn-flakes au visage ; Véronique est alors livrée à elle-même.

Le film reste cependant plus ambigu sur la représentation de Coluche en mauvais père que ce que cette scène peut laisser penser, c'est-à-dire que Coluche est moins un père absent en lui-même qu'il ne le devient, parce qu'il est corrompu par la course au pouvoir. Plus tôt dans le film, une scène présente Coluche avec ses enfants dans leur chambre, où il prend le temps de leur raconter une histoire drôle pour qu'ils s'endorment. Le statut paternel de Coluche est donc moins un état qu'une évolution qui marque son aveuglement progressif vis-à-vis du pouvoir qu'il convoite. Le paradoxe est alors le suivant : plus Coluche souhaite le pouvoir politique, plus il se désintéresse du pouvoir familial. Il substitue à sa famille les marginaux qu'il entend représenter (selon son célèbre discours) ${ }^{11}$.

Ce désir d'être un père pour «tous ceux qui ne comptent pas pour les hommes politiques $^{12}$ »- désir mis en scène dans une séquence où le discours de Coluche fait écho dans tous les foyers français par l'intermédiaire de la télévision (avec ce long travelling truqué qui nous fait passer d'un décor à un autre), comme si, grâce à elle, il prenait alors la tête de chaque groupe et de chaque foyer - l'isole de la possibilité directe que lui offrent ses enfants d'être un bon père, et se mue en une question problématique : comment un homme qui ne peut même pas s'occuper correctement de ses enfants pourrait-il devenir le « chef de la famille française »? 
31 Ainsi, c'est sa soif de pouvoir qui le décrédibilise aux yeux des siens, et plus largement aux nôtres. Coluche en mauvais père ne peut pas réellement aspirer au pouvoir politique puisque les deux, père et président, sont assimilés. Paradoxalement, c'est bien la conquête du pouvoir qui en fait un mauvais père. La soif de pouvoir est donc opposée au pouvoir naturel, au pouvoir du chef de famille, comme si la conquête du pouvoir devait corrompre le pouvoir légitime du « $\operatorname{chef}^{13}$ ".

En ce sens, le schéma paternel est à la fois affirmé et infirmé. Affirmé car Coluche propose une vision naturalisée du pouvoir, un pouvoir qui échoit d'en haut en assimilant le père à la fonction présidentielle (un bon père est un bon chef). Infirmé car justement, en étant naturel, ce pouvoir ne peut se conquérir (la conquête est une envie de pouvoir), or le jeu électoral suppose que le politicien désire le pouvoir pour l'avoir ${ }^{14}$. L'ambiguïté de Coluche résulte alors de cette tension entre un pouvoir qui ne se conquiert pas (naturel) et l'affirmation de la figure paternelle comme prérequis d'un bon modèle politique. Ainsi, être le «chef de la famille française» suppose une légitimité qui ne soit pas démocratique, qui, plus encore, est démentie par le jeu démocratique de la conquête du pouvoir. De fait, la représentation du père dans les films s'accompagne d'une démythification constante, d'une banalisation, d'un retour à la normalité pour le corps de l'homme politique que la fonction présidentielle excède.

\section{Le père déchu}

Certains films du corpus n'isolent pas la cellule familiale pour l'élever au rang de symbole du pouvoir, mais créent entre deux personnages une relation de domination qu'ils assimilent souvent à celle d'un père et de son fils. À la différence de La Conquête et de Coluche, les films suivants élaborent une relation de substitut filial à l'intérieur même du cercle politique, dans le cadre public et non plus dans le cadre privé ${ }^{15}$. S'il est possible de parler de relation filiale, c'est parce que les films sont, en sous-texte, des récits d'initiation dans lesquels les personnages néophytes font l'expérience du monde politique qu'ils ne connaissaient pas avant ${ }^{16}$. Pourtant, comme dans la majorité des récits d'initiation, la figure tutélaire est in fine remise en cause et ramenée au banal, à une faiblesse humaine. En d'autres termes, elle se trouve désacralisée.

\section{La figure du mal}

Dans L'Exercice de l'État, Bertrand Saint-Jean (Olivier Gourmet) est un nouveau venu dans le paysage politique; il est issu du monde ouvrier et animé par une foi en son travail qui s'émoussera au fil du film. L'Exercice de l'État est l'exemple d'une initiation douloureuse, où l'apprentissage du travail politique équivaut au sacrifice de ses idéaux.

La figure paternelle est désignée explicitement sur un mode quasi parodique puisque les personnages surnomment le président le «P.R.» (on prononce ces initiales de "Président de la République » comme " père »). Ce chef de famille politique, le chef du gouvernement, n'apparaît que deux fois dans le film et seulement une fois avec SaintJean, à la fin, quand il l'affecte à un nouveau ministère. Cette absence physique d'un président dont le sigle reste pourtant présent sur toutes les lèvres favorise la dimension sacrée du personnage, comme si le nommer suffisait à figurer l'ampleur de son aura. De fait, il est avant tout représenté comme un monarque plutôt que comme un président, 
bien moins soucieux du bien commun que de l'établissement de son propre pouvoir. Lors de sa première apparition, il est assis seul au milieu d'un grand canapé qu'il occupe avec le confort d'un homme sûr de son pouvoir, quand ses ministres sont sur des chaises, souvent filmés à deux dans un cadre qui les étouffe. Le P. R. semble alors le souverain du champ. Si le corps présidentiel est absent ou souverain, celui de SaintJean est au contraire ramené à sa corporéité essentielle, puisqu'on le voit durant le film saoul, faire l'amour, courir, souffler. Olivier Gourmet appuie énormément sur la dimension physique de son personnage, ce qui accentue d'autant plus la césure entre le corps présidentiel et celui de ses subalternes. La dimension sacrée du président est largement mise en valeur dans L'Exercice de l'État, mais celle-ci est négative. Dans la confrontation entre Saint-Jean et le président (qui n'a d'ailleurs aucun nom), le dialogue se charge de caractériser ce dernier comme un tyran, ou plus exactement, ce qu'il dit lui-même, comme "un dieu ${ }^{17} »$. Saint-Jean doit abandonner ses idéaux pour " désamorcer, désamorcer, désamorcer » et finalement « récupérer les cinq points dans les sondages que vont faire perdre la privatisation des gares » (répliques du président). L'initiation à la politique se joue pour Saint-Jean par l'intermédiaire de ce père diabolique et désenchanté qui le fait en quelque sorte entrer dans le moule, entrer dans le monde étriqué et privé des politiciens, des calculateurs et des carriéristes. Le père de L'Exercice de l'État, le président, apprend à Saint-Jean à perdre tout sens du politique ; la sacralisation du père est donc perçue comme la simple déférence d'un subalterne envers une figure du mal.

\section{La parodie}

Dans Quai d'Orsay, la figure paternelle est très rapidement mise en évidence pour finalement être, sinon moquée, au moins largement compromise.

La première rencontre entre Arthur (Raphaël Personnaz) et le ministre (Alexandre Taillard de Worms joué par Thierry L'Hermitte) se fonde sur la mise en place des stéréotypes qui préparent l'arrivée de la figure paternelle: faste des lieux contre petitesse du jeune homme, cérémonial contre naturel ; soit la fascination d'un ingénu envers un monde qu'il découvre. Cette fascination est la même à la rencontre du ministre, vif, énergique, ambitieux, grandiloquent, dans lequel Antoine avoue avec légèreté reconnaître un père ${ }^{18}$.

Quai d'Orsay est un film qui a intégré en lui tous les poncifs traditionnels de la représentation politique et qui les emploie avec cette même légèreté, conscient qu'une certaine forme d'évidence entoure ces différentes figures dont la plus évidente de toutes est celle du père. La parodie réside dans le constat expéditif d'Arthur sur la qualité de père spirituel du ministre, comme s'il n'existait là qu'un processus initiatique banal.

Quai d'Orsay s'emploie donc le plus rapidement possible à rendre cette figure décevante, voire ridicule et à reporter le récit sur le travail politique, tout en entretenant un certain flou sur le ministre Taillard (est-ce un génie ou est-il simplement stupide ?). La figure paternelle, si elle est convoquée explicitement dès la première rencontre entre les deux personnages, ne survit pas au traitement ironique qu'en fait le film, la véritable figure tutélaire étant plutôt incarnée par le directeur de cabinet Mauppa (Niels Arestrup) que par le ministre. 

Vincent Lindon joue un jeune chef d'entreprise auquel le président de la République demande de devenir le Premier ministre de la France. Il est donc, lui aussi, un néophyte qui entre en carrière politique sans en avoir l'habitus ${ }^{19}$. Le Premier ministre est donc dans une situation initiatique puisqu'il doit apprendre à devenir un politicien; sa position hiérarchique inférieure se double d'une infériorité symbolique liée à son manque d'expérience, il devient alors un élève par rapport à Alain Cavalier.

La richesse de Pater tient dans la résonance de ce rapport filial symbolique à tous les niveaux du film. C'est autant la relation entre un président et son Premier ministre, qu'entre un réalisateur et son acteur ou entre deux hommes de générations différentes : des liens à la fois politiques, professionnels et personnels, sans que ceux-ci ne trouvent réellement leur indépendance, puisque le dispositif filmique tend à entremêler ce qui relève ou non du jeu.

Du point de vue de la fiction politique que Pater met en place, le film reprend l'idée classique du fils qui tue le père, ce qui se matérialise dans le récit par la candidature de Vincent Lindon aux présidentielles contre Alain Cavalier. La figure paternelle censée être incarnée par Alain Cavalier s'en trouve certes dépassée mais aussi confirmée : s'il faut tuer le père, c'est bien que celui-ci existe.

Plus profondément, Pater nie la réalité même de son titre par le dispositif filmique qu'il met en place. Si les positions fictionnelles et sociales entretiennent les rapports de domination (président / Premier ministre, réalisateur / acteur, vieux / plus jeune), la mise en scène dément constamment cette hiérarchie. Il ne s'agit pas de créer une distance, de former entre les deux hommes un écart qui pourrait s'investir d'une sacralité (donc de réaffirmer l'idée du père que promet le titre), mais plutôt de rapprocher, d'unir, de lier et finalement d'abolir, dans l'échange de pouvoir entre celui qui filme et celui qui est filmé, dans leur façon de partager la caméra, les différences qui devraient les séparer.

45 La dernière scène est en ce sens remarquable puisqu'elle met au jour le dispositif filmique, à savoir que chacun possède une caméra pour filmer l'autre, et met en abîme ce procédé quand Vincent Lindon propose à Alain Cavalier une idée de jeu et de montage que le réalisateur reproduit ensuite dans son film (un champ/contrechamp avec un raccord dans le mouvement). Le geste est purement égalitaire et ramène la légitimité hiérarchique à ce qu'elle est : une fiction qui s'alimente d'elle-même et qu'on nourrit de symboles, de sens et de valeur pour la rendre plus réelle. C'est bien ce qui fait de Pater une parodie puisque, loin de réaffirmer les rapports de force dits « légitimes » que la société met en place, il les annihile et dévoile l'illusion profonde qui les soutient (ils n'existent que par croyance en leur légitimité).

Ainsi, Pater semble nier la proposition avancée par son titre : il y a moins un père que le fils vient sacraliser qu'une déconstruction de ce modèle comme la promesse d'un retour vers une relation de parité, une relation humaine.

\section{Conclusion}

Que retirer de cette distinction entre des films politiques familiaux d'un côté (qui mettent en scène une vie privée), et initiatiques de l'autre (mise en scène d'un regard filial symbolique)? On voit d'abord que certains films (La Conquête, Coluche, Le Candidat), 
par cette caractérisation du politicien dans le cercle privé réaffirment la prégnance de la figure paternelle en politique, par le négatif (Coluche comme mauvais père, La Conquête comme mauvais mari) ou le positif (Le Candidat qui devient un père en récupérant sa femme et en échappant à la tutelle de ses mentors). Ces films apposent sur l'univers politique la forme d'une sacralité familiale classique et réactivent l'imaginaire réactionnaire du pouvoir français (monarchique et masculin). Ainsi, ils se font l'écho de la méfiance que peuvent inspirer les élus nationaux, du fait même de cette incapacité à incarner la fonction politique. La figure paternelle est un échec car les hommes ne parviennent pas à atteindre la grandeur de la fonction, mais elle reste donc le modèle visé, voire idéal.

D'autres films peuvent se lire comme des récits initiatiques et le cercle privé ne renvoie pas implicitement à la qualité politique des personnages. Plus encore, le cercle privé est souvent vierge de figure paternelle, celle-ci ne se retrouvant que par substitut dans les relations de travail. En vérité, dans ces films (L'Exercice de l'État, Quai d'Orsay, Pater), la figure du père est un simple échelon narratif qui ne sert que plus efficacement le récit d'initiation. C'est-à-dire que la sacralité qu'on accorde à la figure se restreint aux relations que tissent les personnages, et non à celles d'un président et de ses citoyens. Plus encore, cette figure n'est que rarement un aboutissement et elle est souvent dépassée (Pater, Quai d'Orsay) comme s'il fallait bien que le charme de son illusion s'efface pour laisser place à la réalité humaine. Le père s'en trouve banalisé, normalisé et, enfin, détaché de cette fonction qu'il ne peut vraiment incarner.

Finalement, il semble que la fonction présidentielle soit investie d'une sacralité et d'une ampleur que l'homme politique ne peut vraiment atteindre, comme si les attentes étaient trop fortes et qu'il devait subir les contradictions de ce qu'implique la fonction sans pouvoir les résoudre. En un sens, le président ne peut se détacher de sa propre corporéité qui le réduit au statut d'homme et l'éloigne de l'idée présidentielle.

Un film comme Coluche va plus loin en laissant entendre que le système démocratique lui-même, en tant qu'il ne naturalise pas le pouvoir (sa légitimité n'est pas naturelle) et qu'il faut avant tout le vouloir pour l'obtenir, crée un paradoxe indépassable entre la figure paternelle (naturelle, légitime) liée à la fonction présidentielle et l'impossibilité qu'offre le système d'exercer un pouvoir légitime. C'est un peu comme si l'idéal de la fonction politique ne s'adaptait pas à la réalité de son système; en d'autres termes, comme si à un pouvoir démocratique (incarné par l'homme) on associait un idéal monarchique (la fonction). On peut noter à quel point cette vision dénote de «l'empreinte de la pratique gaullienne sur l'institution présidentielle» dont "l'héritage s'est avéré le plus souvent complexe, voire impossible » dans la mesure où le poste présidentiel s'avère être "une robe beaucoup trop lourde à porter pour quiconque ici-bas » (Achin $2008: 22$ ).

51 La figure paternelle est alors un reste de cet idéal monarchique (pouvoir légitime et naturel) qu'on accole à la fonction politique par excès. Les films étudiés sont ainsi pris au cœur d'un double mouvement paradoxal: certains valorisent la figure paternelle mais supposent une incapacité de l'homme politique à l'incarner (La Conquête, Coluche, où, comme dans L'Exercice de l'État, la figure tutélaire est mauvaise, quasi maléfique), d'autres partent du principe que cette figure est insuffisante et la moquent ou la banalisent (Quai d'Orsay, Pater). Ce paradoxe évoque dans les deux cas une perte de sacralité de l'homme politique, soit parce qu'il ne peut incarner l'idéal de la fonction qu'il occupe, soit parce qu'il est ramené à sa qualité humaine au-delà de sa position 
sociale. D'une certaine façon, les films dévoilent les faiblesses de ce double corps de l'homme politique évoqué en introduction. À la fois parce que le corps fictif, imagé, ne répond pas aux attentes de la fonction présidentielle, mais aussi car ce retour aux nécessités humaines est un appel vers un corps réel, physique, qui est désacralisé. Il semble que la représentation politique, donc la politique elle-même, soit à un tournant où la légitimité du pouvoir, puisqu'elle ne s'appuie plus sur une sacralité traditionnelle, doive s'affirmer par d'autres moyens. Il est possible que cette conjonction importante de films sur la politique soit aussi le reflet d'un malaise profond sur la sacralité et la représentation politique, comme s'il fallait soit se défaire des attentes de la sacralité (hauteur de la fonction) soit lui trouver un nouveau terrain d'expression.

\section{BIBLIOGRAPHIE}

Achin, Catherine et Dorlin, Elsa, 2008, « Nicolas Sarkozy ou la masculinité mascarade du Président », Raisons politiques, $\mathrm{n}^{\circ} 31$, p. 19-45, [En ligne], https://www.cairn.info/revue-raisonspolitiques-2008-3-page-19.htm, consulté le 27 novembre 2019.

DOI : $10.3917 /$ rai.031.0019

Anese, Franck, Barbier, Joachim, Beaugé, Marc et Régy, Stéphane, 2015, « Interview de François Hollande ", Society.

Déhée, Yannick, 2000, Mythologie politique du cinéma français 1960-2000, Paris, PUF.

Gauchet, Marcel, 2007, « Le politique versus la politique », Le blog Marcel Gauchet, [En ligne], http://gauchet.blogspot.com/2007/11/le-politique-versus-la-politique.html, consulté le 27 novembre 2019.

Girardet, Raoul, 1986, Mythes et mythologies politiques, Paris, Seuil.

Kott, Sandrine et Michonneau, Stéphane, 2006, Dictionnaire des nations et des nationalismes, Paris, Hatier.

Liatard, Séverine, 2008, Les Femmes politiques en France, de 1945 à nos jours, Paris, Éditions complexes.

Mandelbaum, Jacques et Rauger, Jean-François, 2005, « Le cinéma et la politique en concurrence », Le Monde, 19 février 2005, [En ligne], https://www.lemonde.fr/idees/article/ 2005/02/19/le-cinema-et-la-politique-en-concurrence_398783_3232.html, consulté le 27 novembre 2019.

Marin, Louis, 1981, Le Portrait du roi, Paris, Minuit.

Rancière, Jacques, 2005, La Haine de la démocratie, Paris, La fabrique.

Sellier, Geneviève, 2012, « Images du pouvoir politique dans le cinéma français contemporain », Paris, Conférence inédite au Forum des images.

Verjus, Anne, 2013, « La paternité au fil de l'histoire », Informations Sociales, n 176, p. 14-22. 


\section{Corpus}

Arestrup, Niels, 2006, Le Candidat, France, 95 minutes.

Caunes, Antoine de, 2008, Coluche l'histoire d'un mec, France, 100 minutes.

Cavalier, Alain, 2011, Pater, France, 105 minutes.

Durringer, Xavier, 2011, La Conquête, France, 105 minutes.

Guédiguian, Robert, 2005, Le Promeneur du Champ-de-Mars, France, 117 minutes.

Schoeller, Pierre, 2011, L'Exercice de l'État, 115 minutes.

Vincent, Christian, 2012, Les Saveurs du Palais, 95 minutes.

Tavernier, Bertrand, 2013, Quai D'Orsay, 113 minutes.

\section{NOTES}

1. On pourrait ajouter que dans le cinéma français, la politique est souvent restée du côté de la comédie. Des années 1930 aux années 1950, les comédies françaises inspirées par la tradition du guignol tendent à ridiculiser tout personnage de pouvoir, même à plus petite échelle, tel que les maires de village. Comme le notent Jacques Mandelbaum et Jean-François Rauger (2005: 11) : "les années 1930 ont été riches en figures de politiciens cocasses campées selon les prescriptions d'un antiparlementarisme fort en vogue à l'époque ».

2. Si le terme de "politicien " est aujourd'hui perçu comme péjoratif, il faut bien l'entendre ici au sens strict, c'est-à-dire comme désignant simplement un homme qui fait de la politique.

3. Si le père n'est pas nécessairement l'enjeu central de l'ensemble des films, il se retrouve en filigrane, d'une façon ou d'une autre, à travers les différentes dramaturgies qui seront détaillées au fil de l'article.

4. Cette double identité de la masculinité nourrie des représentations genrées de la nation dans lesquelles la femme incarne des symboles de natalité, mais fonde aussi une pratique de l'exercice du pouvoir qui « demeure essentiellement masculin » (Kott 2006 : 160).

5. Ces expressions viennent désigner une forme d'exercice du politique qu'il nomme d'après le terme latin gravitas.

6. Exemplairement Staline, considéré comme « le petit Père des peuples ».

7. Il cite le maréchal Pétain qui dit vouloir « rebâtir la maison France » ou "restaurer l'ordre dans la maison » (Girardet 1986 : 74).

8. Si sa lecture s'applique avant tout au monarque, on peut l'étendre à la représentation de tout homme de pouvoir, surtout le président, dont le corps se dédouble lui aussi dans de nombreuses images officielles, comme la photo présidentielle, sorte de portrait du roi contemporain.

9. On retrouve là la fonction classique du corps féminin dans les représentations politiques. "Le corps féminin, de par sa fonction traditionnelle de personnification symbolique de la Nation, de la Patrie, de la République, etc., est toujours apparu comme 
un signifiant du politique et de ses valeurs, un corps "allégorique". Ainsi, la féminité renvoyait toujours à autre chose qu'à elle-même » (Achin $2008: 35$ ).

10. Dans Pater c'est un homme d'entreprise ; dans Quai d'Orsay un jeune communicant ; dans L'Exercice de l'État Saint-Jean est un nouveau dans le paysage politique et vient du monde ouvrier.

11. «J'appelle les fainéants, les crasseux, les drogués, les alcooliques, les pédés, les femmes, les parasites, les jeunes, les vieux, les artistes, les taulards, les gouines, les apprentis, les Noirs, les piétons, les Arabes, les Français, les chevelus, les fous, les travestis, les anciens communistes, les abstentionnistes convaincus, tous ceux qui ne comptent pas pour les hommes politiques à voter pour moi, à s'inscrire dans leurs mairies et à colporter la nouvelle. »

12. Tiré de son discours de candidature que le film réutilise.

13. On retrouve là les traces d'une vision ancienne du politique dont on trouve les racines dans la démocratie athénienne telle que Platon l'envisage et que Jacques Rancière (2005: 50) réactualise en l'exprimant de cette façon : « Le bon gouvernement c'est le gouvernement de ceux qui ne désirent pas gouverner " (faisant référence au système de tirage au sort pour les élections athéniennes).

14. On peut se référer ici à la distinction qu'effectue Marcel Gauchet entre le politique et la politique. Pour lui, le politique est un héritage de la monarchie ou, plus largement, de tout système dans lequel le pouvoir « tombe du ciel », c'est-à-dire où il est constitué par une légitimité religieuse, voire divine. À l'inverse, la politique est d'une nature beaucoup plus récente et serait l'invention du " pouvoir par représentation » (Gauchet 2007). La question que se pose Marcel Gauchet est donc de savoir si la politique a définitivement écrasé le politique : «est-ce que quelque chose de l'ancien politique, qui s'imposait avec une légitimité religieuse ou traditionnelle, ne survit pas à l'intérieur de la politique ?» (Gauchet 2007). On peut se demander à quel point la figure du père n'est justement pas le reliquat d'une "légitimité traditionnelle" qui persiste dans les représentations actuelles du pouvoir.

15. C'est en fait souvent une relation à la fois de domination hiérarchique et de fascination envers celui qui détient et connaît le pouvoir.

16. Coluche n'est pas un récit initiatique, c'est même tout le contraire puisque le personnage ne parvient jamais à pénétrer le monde politique ; il ne fait ainsi que l'apprentissage de l'impossibilité d'apprendre de ce monde qu'il ne pourra jamais fréquenter.

17. «Je te parle des cadeaux que les dieux mettent sur nos chemins, si tu ne les utilises pas, les dieux peuvent se venger. »

18. "Ça c'est le truc des mecs qui ont pas eu de père. »

19. C'est à nuancer puisqu'il nous est dit que Vincent Lindon a déjà été député une fois (disons qu'il est néophyte à un poste aussi important). 


\section{RÉSUMÉS}

Depuis la fin du XIx ${ }^{e}$ siècle, la nation produit un imaginaire vivace dont l'illusion tient à la dimension a-historique qu'on lui confère, comme si elle existait de toute éternité. À cet égard, les figurations du pouvoir national sont aussi importantes que son action réelle. La figure du père en tant que souverain aimant, protecteur, sécurisant, est l'une des images les plus éculées des propagandes politiques (les grands dictateurs sont souvent des pères pour la nation). Ainsi l'un des attributs masculins modélisant le plus synthétiquement ce que le peuple peut attendre d'un politicien semble-t-il être celui de la paternité.

Cet article se propose de questionner la représentation récente de la figure paternelle dans les films présentant des politiciens. Le corpus proposé est le suivant : Le Promeneur du Champ-de-Mars (Robert Guédiguian, 2005), Le Candidat (Niels Arestrup, 2006), Coluche l'histoire d'un mec (Antoine de Caunes, 2008), La Conquête (Xavier Durringer, 2011), Pater (Alain Cavalier, 2011), L'Exercice de l'État (Pierre Schoeller, 2011), Les Saveurs du Palais (Christian Vincent, 2012) et Quai d'Orsay (Bertrand Tavernier, 2013). L'objectif sera de révéler la persistance d'une représentation du père en politique, tout en discutant l'ambivalence qui conduit certains films à reproduire l'analogie entre bon père et bon chef quand d'autres la discutent, la moquent, pour en présenter l'inanité.

Since the end of the nineteenth century, the nation produces a long-lived imaginary of whom the illusion holds thanks to the a-historical dimension we confer to it, as if it existed from time immemorial. In that regard, the characterizations of its political power are as important as its genuine actions. The Father figure as a loving, protective, and reassuring sovereign is political propagandas' most worn off icons (great dictators are often Fathers for, and of, the nation). Thereby, the male attribute which most concisely modelizes what the people may expect from a politician is that of paternity.

Recently, in French films focusing on politicians (as characters), this paternalistic postulate evicts almost systematically female characters from political representation. Women are invisible (Pater), or characterizing the male figure (in domestic boundaries), or even serving the benefit of his public image (within professional boundaries). They are never the bearers of power. The absence of a female representation of power questions on the persistence of this paternal ideal, which we associate to national political power.

In the films under scrutiny, representing political power representation by the Father figure may be read in two ways: on the one hand, some films present a settled politician, both in the private and public sphere (Coluche, La Conquete, Le Candidat); on the other hand, some films show a character as a beginner in the political world, in search of tutelary figures (L'Exercice de l'État, Pater, Quai d'Orsay). The former films tend to show the character as a paternal figure in his inner circle, in domestic boundaries: they use relationships between characters belonging to his domestic circle (father/children, husband/wife) in order to properly craft a father figure which is supposed to expand itself outside of his private sphere (into the public sphere). In other words, a good father portrays a good statesman; a bad father, a bad leader. On the contrary, films which use an initiatory logic to represent the father figure do not characterize the latter by his private sphere but rather by his position of a point of contact regarding a new comer in the political world. Here, the paternal figure is therefore not revealed straightaway, neither is it associated to the private sphere. The figure is rather a mere fact that is constructed in the course of the initiation story, and that films often tend to mock.

This article aims a questioning endeavours to question the paternal figure's recent representation in French films focusing on politicians as characters. The film corpus used in this study is as follows: Le Promeneur du Champ-de-Mars (Robert Guédiguian, 2005), Le Candidat (Niels 
Arestrup, 2006), Coluche l'histoire d'un mec (Antoine de Caunes, 2008), La Conquête (Xavier Durringer, 2011), Pater (Alain Cavalier, 2011), L'Exercice de l'État (Pierre Schoeller, 2011), Les Saveurs du Palais (Christian Vincent, 2012), Quai d'Orsay (Bertrand Tavernier, 2013) et Gaz de France (Benoît Forgeard, 2015). The goal will be to reveal the persistence of a representation of paternity in politics, while discussing the ambivalence aforementioned: some films renew the analogy between "good father" and "good leader," some put it to a test, they mock it, to show its futility.

\section{INDEX}

Keywords : politician, cinema, French cinema, nation, patriarchy, representation

Mots-clés : politicien, cinéma, cinéma français, nation, patriarcat, représentation

\section{AUTEUR}

\section{ROBIN HOPQUIN}

Université Paris 1 Panthéon Sorbonne 